\title{
Elucidating the mechanism of miRNA-214 in the regulation of gingival carcinoma
}

\author{
YU GONG $^{1}$, HONGLI YANG ${ }^{1}$ and XIN TIAN ${ }^{2}$ \\ ${ }^{1}$ Emergency Department; ${ }^{2}$ Department of Oral Pathology, Affiliated Stomatology Hospital of Kunming Medical University, \\ Kunming, Yunnan 650031, P.R. China
}

Received November 6, 2015; Accepted December 9, 2016

DOI: $10.3892 /$ etm.2017.4264

\begin{abstract}
The aim of the present study was to evaluate the expression levels of microRNA (miRNA)-214 in tumor tissue, blood and saliva of patients with gingival carcinoma, and to investigate the mechanisms underlying the infiltration and invasion of gingival carcinoma. Between January 2013 and March 2015, blood and saliva samples, gingival carcinoma tumor specimens and peritumoral tumor tissues were harvested from 56 patients with gingival carcinoma. Blood and saliva samples were also harvested from 33 control patients without gingival carcinoma. Reverse transcription-quantitative polymerase chain reaction (RT-qPCR) was performed to detect miRNA-214 and protein tyrosine phosphatase gene (PTEN) mRNA levels. Western blotting and ELISA were performed to detect PTEN protein levels. The results of RT-qPCR demonstrated that the expression of PTEN mRNA in tumor tissues, blood and saliva of patients with gingival carcinoma were significantly decreased compared with that of the control group $(\mathrm{P}<0.05)$. These findings were consistent were consistent with the results of PTEN protein expression detected via western blotting and ELISA in these samples $(\mathrm{P}<0.05)$. Conversely, the expression levels of miRNA-214 in these samples were significantly increased $(\mathrm{P}<0.05)$ in patients with gingival carcinoma compared with the control group. The decreased expression of PTEN may be associated with the expression of miRNA-214. miRNA-214 may regulate infiltration and invasion of gingival carcinoma via PTEN. These results suggest that miRNA-214 may be used as a marker of gingival carcinoma.
\end{abstract}

\section{Introduction}

The majority of gingival tumors are highly differentiated squamous cell carcinomas, such as oral squamous cell

Correspondence to: $\mathrm{Dr} \mathrm{Yu}$ Gong, Emergency Department, Affiliated Stomatology Hospital of Kunming Medical University, 1088 Haiyanzhong Road, Kunming, Yunnan 650031, P.R. China E-mail: ygong01@126.com

Key words: microRNA-214, gingival carcinoma, protein tyrosine phosphatase gene carcinoma (OSCC) (1). These tumors typically grow slowly and present as ulcers (2). In early stages, tumors are able to infiltrate mandibular alveoli, leading to bone destruction, tooth mobility and pain (1). Surgical therapy is recommended for the treatment for OSCC; however, due to the high degree of malignancy, advanced tumors typically infiltrate surrounding tissues or migrate distantly (3). Migration, which is the leading cause of mortality in patients with gingival carcinoma, occurs in $>30 \%$ of patients with negative lymph node diagnoses (4). Surgery and chemotherapy are less effective under these circumstances (1). To reduce tumor recurrence and metastasis, and improve survival, $>70 \%$ of patients have undergone radical treatment which is unnecessary and typically leads to adverse complications, such as severe facial deformities and swallowing, speech, neck and shoulder dysfunction (5-7). Therefore, the identification of accurate diagnostic markers and novel therapeutic targets of gingival carcinoma is necessary.

At present, the activation of oncogenes and mutations of tumor suppressor genes are believed to be the primary mechanisms underlying tumorigenesis $(8,9)$. Similarly, the interactions and coinciding actions of various pathways may result in the tumorigenesis and progression of gingival carcinoma; a process with which various mRNAs and micro (mi)RNAs are associated $(10,11)$. Various recent studies have demonstrated that miRNA-214 is associated with tumorigenesis and progression. For example, Zhang et al (12) showed that miRNA-214 may be used as a diagnostic predictor of gastric tumor and reduce tumor cell proliferation and invasion. Wang et al (13) demonstrated that miRNA-214 was able to modulate the proliferation and invasion of breast cancer via tumour protein p53 (p53). Wang et al (13) reported that miRNA-214 had a suppressive role in bladder cancer via targeting p53 and DNA-damage regulated protein 1 (14). Wan et al (15) demonstrated that miRNA-214 was able to protect cardiomyocytes in follow-up treatment of myocardial ischemia. Izawa et al (16) reported that miRNA-214 may prevent liver fibrosis and effectively relieve liver cirrhosis induced by thioacetamide. These findings suggest that miRNA-214 may be a novel therapeutic agent for cancer prevention, diagnosis and treatment.

Protein tyrosine phosphatase gene (PTEN) belongs to the tumor suppressor gene family. Mutation of PTEN is associated with the development of various human malignancies, and has key roles in apoptosis, cell cycle and cell migration (17-23). In various malignancies, such as prostate cancer (24), brain 
tumor (6), endometrial cancer (25), glioblastoma (26), melanoma (27), cervical cancer (28), colorectal gland cancer (29), breast cancer (30) and colon cancer (31) PTEN is downregulated. Furthermore, PTEN is associated with gingival carcinoma and its expression is decreased in gingival tumor tissues (10). Another recent study has indicated that PTEN is associated with the occurrence, development and metastasis of gingival carcinoma (32). However, the up-stream regulation factor of PTEN in gingival carcinoma remains to be identified.

In the present study, reverse transcription-quantitative polymerase chain reaction (RT-qPCR), western blot analysis, gene bioinformatics prediction and ELISA techniques were performed to evaluate the mRNA and protein expression of PTEN and miRNA-214 levels in the tumor tissues, blood and saliva of patients with gingival carcinoma. The association between PTEN and miRNA-214 was subsequently analyzed.

\section{Materials and methods}

Research subjects and selection criteria. Between January 2013 and March 2015, blood and saliva samples were harvested from 56 gingival carcinoma patients in the Affiliated Stomatology Hospital of Kunming Medical University (Kunming, China). These patients were diagnosed with gingival carcinoma and chose to receive surgical resection treatment. As a control, blood and saliva samples were harvested from 33 patients from the same hospital diagnosed with non-neoplastic gum disease during the same period. Tumor tissues and peritumoral tissues were subsequently harvested from the 56 gingival carcinoma patients. The gingival carcinoma patients included 38 males and 18 females, aged 17-75 years old, with a median age of 58.6 years. The control patients, included 20 males and 13 females, aged 25-72 years old, with a median age of 59.5 years. All patients were at the early stages of gingival carcinoma or non-neoplastic gum disease and were not treated with hormone therapy, traditional medicine, radiotherapy or chemotherapy prior to surgery. The gingival carcinoma patients included 30 patients with gingival carcinoma in the upper gum and 26 patients with gingival carcinoma in the lower gum, and consisted of one case of mucoepidermoid carcinoma, two cases of adenoid cystic carcinoma, two cases of adenocarcinoma, three cases of myoepithelial carcinoma and 48 cases of squamous cell carcinoma. Prior written and informed consent were obtained from all patients and the study was approved by the ethics review board of Affiliated Stomatology Hospital of Kunming Medical University.

Reagents and instruments. The miRcute miRNA isolation kit (dp501), miRcute miRNA first strand cDNA synthesis kit (KR201), miRcute miRNA fluorescent detection kit (FP 401), SuperReal Premix (SYBR Green) (FP 204) and TIANScript II first strand cDNA synthesis kit (KP 107) were purchased from Tiangen Biotech Co., Ltd. (Beijing, China). The PCR-iQ5 system was purchased from Bio-Rad Laboratories, Inc. (Hercules, CA, USA). The polyclonal rabbit anti-human anti-PTEN antibody (ab32199), mouse monoclonal anti- $\beta$-actin antibody (ab6276) and secondary goat anti-rabbit antibody (ab6721) were purchased from Abcam (Cambridge, MA, USA). TRIzol reagent was purchased from Shanghai Yeasen Biotechnology Co., Ltd. (10606ES60; Shanghai, China). The bicinchoninic acid protein assay kit was purchased from Real-Times (Beijing, China) Biotechnology Co., Ltd. (RTP7102; Beijing, China). The miRNeasy serum/plasma kit for serum RNA extraction was purchased from Guangzhou Jianlun Biological Technology Co., Ltd. (JL 217184; Guangzhou, China). The PTEN ELISA kit was purchased from Wuhan USCN Business Co., Ltd. (sE95822Hu; Houston, TX, USA). Image Lab 3.0 software was purchased from Beijing Bio-launching Technologies Co., Ltd. (Beijing, China).

Sample collection. Tumor tissues and peritumoral tissues were preserved in liquid nitrogen for further analysis. Peripheral blood was harvested in the morning following overnight fasting and stored at $-20^{\circ} \mathrm{C}$ following anticoagulation with EDTA.

Prior to saliva collection, research participants were prohibited from food, water and smoking for $2 \mathrm{~h}$ to maintain oral hygiene. To increase the quantity of collected saliva, the head of a sterile cotton swab was moistened with $2 \%$ citric acid solution, and placed on the first sidewall of tongue for $\sim 5$ sec. Following saliva collection, the cotton swab would be applied to the other sidewall. Saliva was collected repeatedly using a sterile enzyme-free centrifuge tube until a total of $5 \mathrm{ml}$ had been harvested.

$R T-q P C R$. The RT-qPCR was carried out according to the manufacturer's protocol. To detect PTEN, an E.Z.N.A. Total DNA/RNA/Protein kit (Omega Bio-Tek, Inc., Norcross, GA, USA) and TRIzol were used according to the manufacturer's protocol to extract total RNA from samples, and RT was performed to produce cDNA (TIANScript II cDNA kit; Tiangen Biotech Co., Ltd., Beijing, China). The total reaction volume was $20 \mu \mathrm{l}$ : $10 \mu \mathrm{l}$ SuperReal Premix (SYBR Green), $0.5 \mu \mathrm{l}$ upstream primers, $0.5 \mu \mathrm{l}$ downstream primers, $2 \mu \mathrm{l}$ cDNA and $7 \mu \mathrm{l}$ double distilled $\mathrm{H}_{2} \mathrm{O}$. Briefly, a 25- $\mu$ 1 RT-qPCR system was performed for 40 cycles according to the following conditions: An initial denaturation was performed at $95^{\circ} \mathrm{C}$ for $3 \mathrm{~min}$, followed by denaturation at $95^{\circ} \mathrm{C}$ for $12 \mathrm{sec}$, annealing at $62^{\circ} \mathrm{C}$ for $40 \mathrm{sec}$, and extension at $72^{\circ} \mathrm{C}$ for $20 \mathrm{sec}$. The results were calculated using $2^{-\Delta \Delta \mathrm{Cq}}$ method (33). The PTEN/ $\beta$-actin ratio was calculated. To detect levels of miRNA-214, RT-qPCR was performed under the same conditions, using U6 as an internal control. The miRNA-214/U6 ratio was calculated. Primers sequences are presented in Table I. Each experiment was repeated three times.

Western blot analysis. Total proteins were extracted and separated by $10 \%$ SDS-PAGE and transferred onto a nitrocellulose membrane. Following blocking with non-fat milk, the membrane was incubated with primary anti-PTEN (1:500) and anti- $\beta$-actin $(1: 5,000)$ antibodies at $4^{\circ} \mathrm{C}$ overnight. The membrane was subsequently washed with TBST 5 times for 5 min. Following washing, the membrane was incubated with goat anti-rabbit secondary antibody $(1: 3,000)$ at room temperature for $1 \mathrm{~h}$. The membrane was subsequently developed using enhanced chemiluminescence plus an enhanced chemiluminescence reagent (BeyonECL Plus; Beyotime Institute of Biotechnology, Haimen, China). Image lab 3.0 software was used to analyze results. $\beta$-actin was used as an internal control. The relative expression level of PTEN was calculated based on the expression of $\beta$-actin. 
ELISA. Blood samples were centrifuged at $1,000 \mathrm{x} \mathrm{g}$ for $10 \mathrm{~min}$ at $4^{\circ} \mathrm{C}$ to separate serum and red blood cells. ELISA was performed according to the manufacturer's protocol of the PTEN ELISA kit. Briefly, blank wells and wells for standard sample, serum and saliva were prepared. In the wells for standard sample, 50- $\mu 1$ standard samples with different concentrations $(180,120,60,30,15,7.5$ and $3.75 \mathrm{pg} / \mathrm{ml})$ were added. In the wells for serum and saliva, $10 \mu \mathrm{l}$ serum or saliva and $40 \mu \mathrm{l}$ dilution solution were added. Nothing was added to blank wells. Besides blank wells, $100 \mu$ l horseradish peroxidase-labeled detection antibody $(1: 1,000)$ mixed with dilution buffer was added to each well. The plate was sealed and incubated for $1 \mathrm{~h}$ at room temperature. Following five repeated washes with the provided washing buffer, the substrate (50 $\mu 1$ substrate A and $50 \mu 1$ substrate B) was added to each well and incubated for $15 \mathrm{~min}$ at $37^{\circ} \mathrm{C}$. The reaction was terminated by adding $50 \mu \mathrm{l}$ stopping solution to each well. Optical density was measured at a wavelength of $450 \mathrm{~nm}$.

Bioinformatics prediction. Bioinformatics prediction is currently the recommended technique for the study of miRNA function (34). In order to evaluate the regulation mechanism of PTEN, miRanda (www.microma.org/rnicroma/home.do), TargetScan (www.targetscan.org), PiTa (www.genie.weizmann. ac.il/pubs/mir07), RNAhybrid (www.hibiserv.techfak.unibielefeld.de/rnahybrid) and Pictar (www.pictar.mdc-berlin.de) programs were used to predict the possible regulatory gene of PTEN.

Statistical analysis. Data were processed using SPSS, version 18.0 (SPSS, Inc., Chicago, IL, USA). Data are presented as the mean \pm standard deviation. The normality of data was tested using the Kolmogorov-Smirnov test. Comparison among multiple sets of measurement data was performed using one-way analysis of variance. When there was homogeneity of variance, least significant difference and Student-Newman-Keuls tests were performed. Otherwise, Tamhane's T2 or Dunnett's T3 method was used. $\mathrm{P}<0.05$ was considered to indicate a statistically significant difference.

\section{Results}

Level of PTEN mRNA in samples. miRNA-214 was identified as one of the genes that may regulate PTEN (Fig. 1). RT-qPCR was performed to detect levels of PTEN mRNA in tumor tissues, peritumoral tissues, blood and saliva. Compared with that in peritumoral tissues, PTEN mRNA levels in tumor tissues of patients with gingival carcinoma was significantly lower $(\mathrm{P}<0.05$; Fig. 2A). Similarly, compared with those observed in control patients, PTEN mRNA levels in the blood and saliva of gingival carcinoma patients were significantly decreased (both $\mathrm{P}<0.05$; Fig. $2 \mathrm{~B}$ and $\mathrm{C}$ ). These results indicate that PTEN may have a role in gingival carcinoma.

Protein expression level of PTEN in tumor and peritumoral tissues. Western blotting was performed to determine the expression level of PTEN in tumor and peritumoral tissues. As demonstrated in Fig. 3, in gingival carcinoma patients, the PTEN protein level in the tumor tissues was significantly decreased compared with that in peritumoral tissues $(\mathrm{P}<0.05)$.
Table I. Primers used in reverse transcription-quantitative polymerase chain reaction.

\begin{tabular}{ll}
\hline Primer & \multicolumn{1}{c}{ Primer sequence } \\
\hline PTEN & \\
Forward & 5'-TTGAAGACCATAACCCACCACAG-3' \\
Reverse & 5'-CATTACACCAGTTCGTCCCTTTC-3' \\
3-actin & \\
Forward & 5'-AAGATGACCCAGATCATGTTTGAGACC-3' \\
Reverse & 5'-GCCAGGTCCAGACGCAGGAT-3' \\
U6 & \\
Forward & 5'-ATTGGAACGATACAGAGAAGATT-3' \\
Reverse & 5'-GGAACGCTTCACGAATTTG-3' \\
miR-214 & \\
Forward & 5'-AGCATAATACAGCAGGCACAGAC-3' \\
Reverse & 5'-AAAGGTTGTTCTCCACTCTCTCAC-3'
\end{tabular}

PTEN, protein tyrosine phosphatase gene; miR, microRNA.

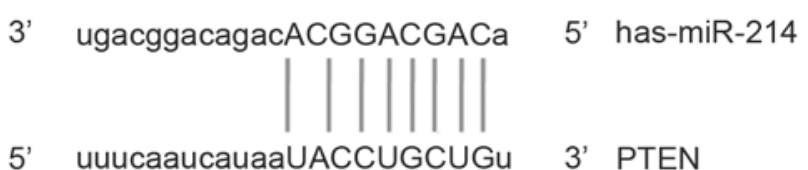

Figure 1. Prediction of the upstream regulatory gene of PTEN. Using miRanda, TargetScan, PiTa, PicTar, and BibiServ, miRNA-214 was predicted as a regulatory gene of PTEN. PTEN, protein tyrosine phosphatase gene; miRNA/miR, microRNA.

This finding was consistent with that observed in PTEN mRNA levels, which suggests that PTEN is downregulated at the transcriptional and protein expression level and that this downregulation may have a role in gingival carcinoma.

Protein expression level of PTEN in blood and saliva. The expression level of PTEN protein in blood and saliva was detected via ELISA. Compared with control patients, the expression level of PTEN was significantly decreased in the blood ( $\mathrm{P}<0.05$; Fig. 4A) and saliva $(\mathrm{P}<0.01$; Fig. 4B) of gingival carcinoma patients. These results were consistent with those observed in PTEN mRNA. As blood is a typical channel of gingival carcinoma metastasis (35), the decrease of PTEN protein in blood may be associated with gingival carcinoma metastasis. Furthermore, the downregulation of PTEN protein level in saliva may be a potential marker of gingival carcinoma.

Expression level of miRNA-214 in tumor tissues, peritumoral tissues, blood and saliva. RT-qPCR was performed to detect expression levels of miRNA-214 in tumor tissues, peritumoral tissues, blood and saliva. As presented in Fig. 5A, miRNA-214 level in the tumor tissues of gingival carcinoma patients was significantly higher than that in peritumoral tissues of gingival carcinoma patients $(\mathrm{P}<0.01)$. Similarly, miRNA-214 levels in the blood (Fig. 5B) and saliva (Fig. 5C) of gingival carcinoma patients were significantly increased in comparison with those in control patients (both $\mathrm{P}<0.01$ ). In combination with the 
A

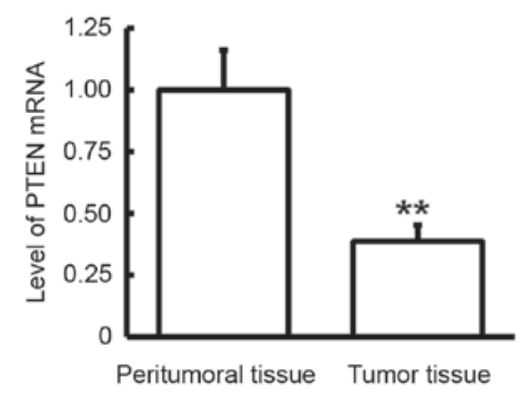

C

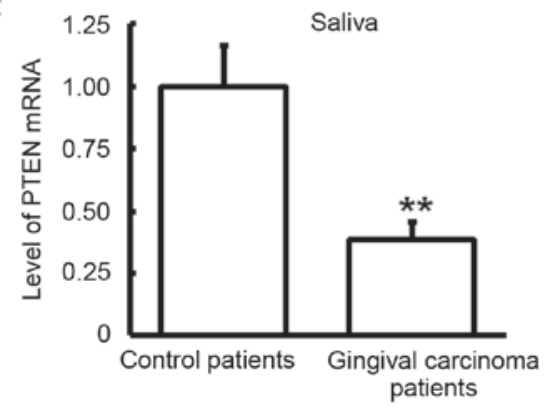

B

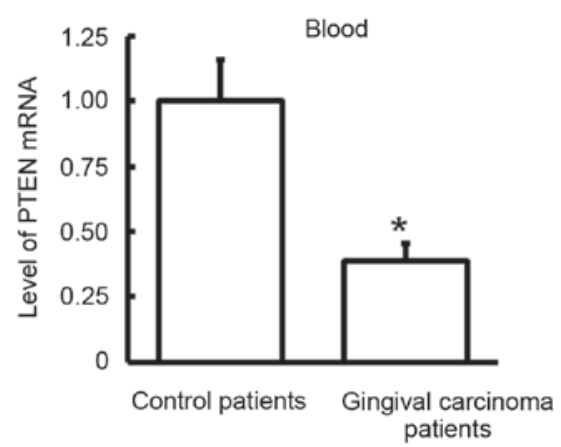

Figure 2. Analysis of PTEN mRNA expression levels. PTEN mRNA expression levels were detected via reverse transcription-quantitative polymerase chain reaction. (A) Expression level of PTEN mRNA in tumor and peritumoral tissues of patients with gingival carcinoma. (B) Expression level of PTEN mRNA in blood of patients with gingival carcinoma and control patients. (C) Expression level of PTEN mRNA in saliva of patients with gingival carcinoma and control patients. ${ }^{*} \mathrm{P}<0.05$ and ${ }^{* *} \mathrm{P}<0.01$. PTEN, protein tyrosine phosphatase gene.

$\mathbf{A}$

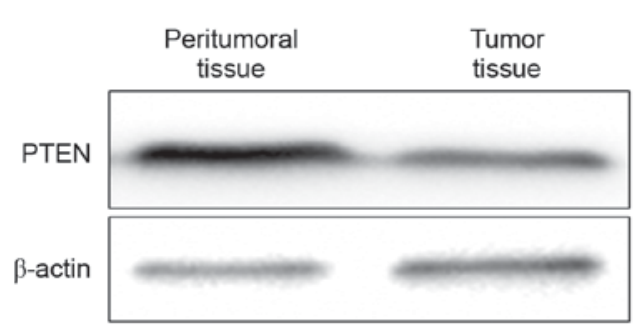

B

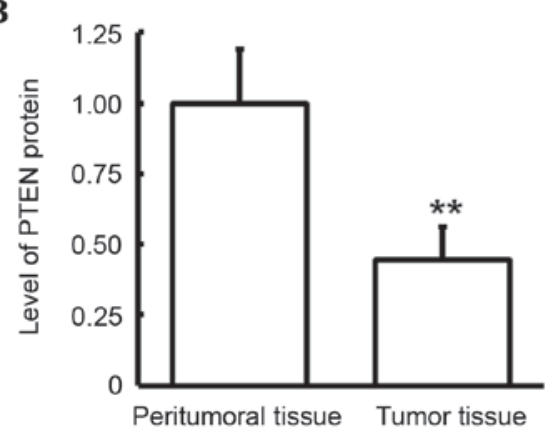

Figure 3. Analysis of PTEN protein expression level in tumor and peritumoral tissues. PTEN protein expression level was detected via western blotting. (A) Representative and (B) quantitative western blot results were presented. ${ }^{* *} \mathrm{P}<0.01$ vs. peritumoral tissues. PTEN, protein tyrosine phosphatase gene.

finding that miRNA-214 may be a regulatory gene PTEN, these results suggest that miRNA-214 may have a regulatory effect on gingival carcinoma, and it may regulate the transcriptional level of PTEN and ultimately affect the protein level of PTEN.

\section{Discussion}

In the present study, the mRNA and protein levels of PTEN in tumor tissues, peritumoral tissues, blood and saliva of patients with gingival carcinoma were evaluated. The expression of miRNA-214, a predicted upstream gene of PTEN, was detected simultaneously, and the potential regulation of PTEN by miRNA-214 in gingival carcinoma was identified.

Among oral and maxillofacial malignancies, $>80 \%$ of tumors are OSCC, and is the sixth most prevalent carcinoma worldwide $(36,37)$. OSCC typically occurs in the tongue, gingiva, and cheek (38). It is clinically characterized by a high degree of malignancy, trend toward regional lymph node metastasis and poor prognosis (2). The majority of advanced OSCCs exhibit invasive growth (39), and it is difficult to surgically remove tumors that have infiltrated surrounding tissues and migrated distantly, and traditional chemotherapy has been demonstrated to have a low efficacy in this scenario (40). The improvement of early diagnosis and the implementation of individualized treatment has become necessary to ameliorate the treatment efficacy and prognosis of OSCC (41). Therefore, there is a need to clarify the pathogenesis of OSCC with surrounding tissue infiltration and distant metastasis and to identify novel gene targets for therapy.

As a tumor suppressor gene, PTEN was discovered in 1997 and is located on chromosome 10 (10q 23.3) $(25,42,43)$. The downregulation or deletion of PTEN has a role in apoptosis, cell cycle and cell migration, and is associated with the development of various human malignancies (44). 
$\mathbf{A}$

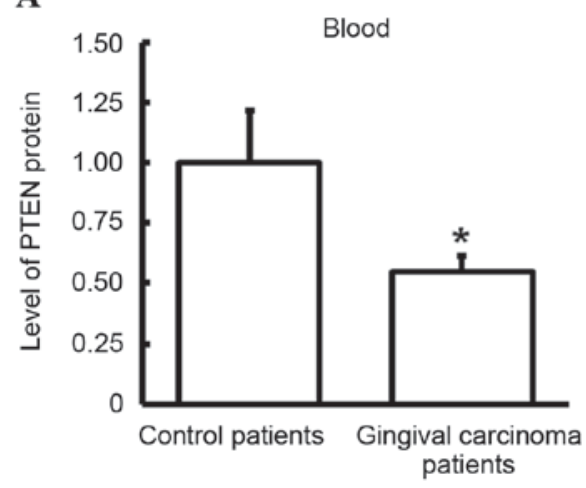

B

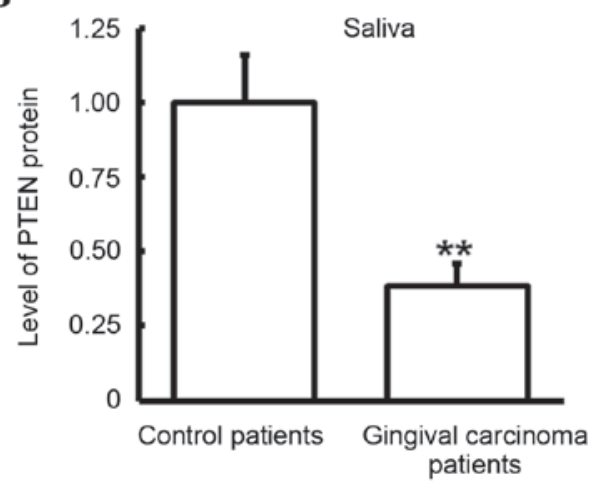

Figure 4. Analysis of PTEN protein expression level in blood and saliva. PTEN protein expression level in blood and saliva was detected using ELISA. (A) Expression level of PTEN protein in blood of patients with gingival carcinoma and control patients. (B) Expression level of PTEN in saliva of patients with gingival carcinoma and control patients. ${ }^{*} \mathrm{P}<0.05$ and ${ }^{* *} \mathrm{P}<0.01$ vs. control patients. PTEN, protein tyrosine phosphatase gene.

A

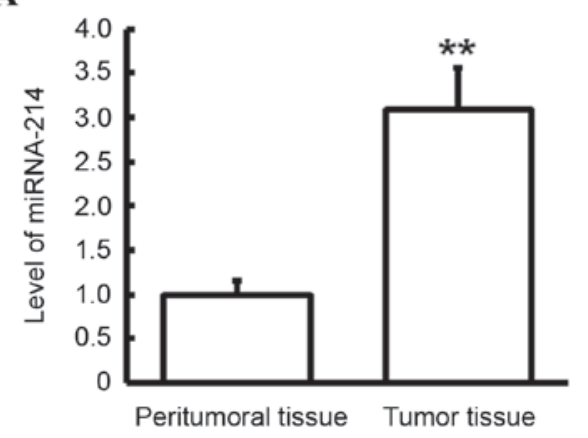

C

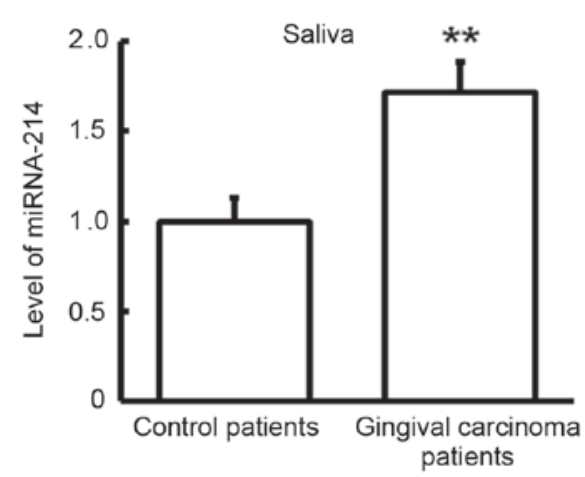

B

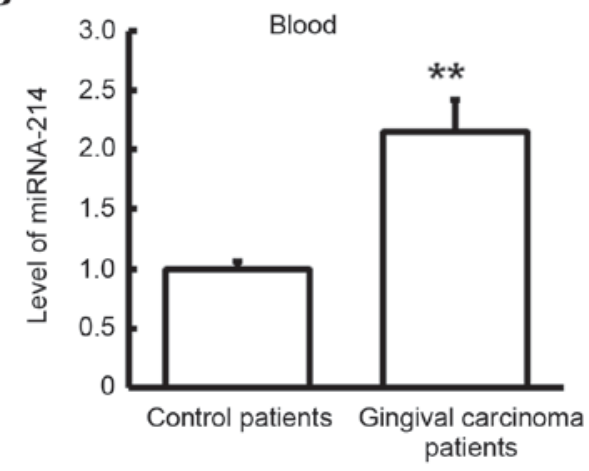

Figure 5. Analysis of miRNA-214 levels. Levels of miRNA-214 were detected via reverse transcription-quantitative polymerase chain reaction. (A) Expression level of miRNA-214 in tumor and peritumoral tissues of patients with gingival carcinoma. (B) Expression level of miRNA-214 in blood of patients with gingival carcinoma and control patients. (C) Expression of miRNA-214 in saliva of patients with gingival carcinoma and control patients. ${ }^{* *} \mathrm{P}<0.01$. miRNA, microRNA.

Previous research on the effect of PTEN on tumorigenesis has predominantly focused on endometrial cancer, glioma, prostate cancer, breast cancer and melanoma (25-31). However, to the best of our knowledge, the role of PTEN in OSCC has been rarely reported. Although the mechanism remains to be elucidated, an abnormal decrease of PTEN has been demonstrated in the majority of types of tumor $(6,25-31)$. In the present study, a significant reduction in PTEN expression was observed in the tumor tissues, blood and saliva of patients with gingival carcinoma compared with control patients. As tumors may migrate through blood as well as via regional invasion, the reduction of PTEN in the blood of patients with gingival carcinoma suggests that PTEN may have a role in tumor metastasis. It is possible that a reduction in PTEN reduces apoptosis, whereas excessive proliferation of cells is associated with increased cell infiltration and mitigation, leading to tumor metastasis. Additionally, changes in PTEN levels in the blood and saliva of patients may be a potential biomarker of gingival carcinoma, particularly in the early stage of tumorigenesis.

Bioinformatics were applied to predict possible upstream regulatory genes of PTEN. It has recently been demonstrated that a class of small, endogenous and non-coding nucleic acid miRNAs are able to prevent transcription of PTEN mRNA (45). According to the current literature, miRNAs have a high level 
of stability in conventional, formalin-fixed, paraffin-embedded clinical tissue specimens, which suggests that they may have potential as diagnostic molecular markers; however, mRNAs tend to be more susceptible to environmental impact and are more unstable than miRNAs (46-48). Furthermore, it has previously been suggested that miRNAs serve important roles in tumorigenesis via their regulation of protein coding genes $(49,50)$. The cleavage of PTEN mRNA may lead to downregulation of PTEN protein. As predicted, miRNA-214 was shown to be closely associated with PTEN; therefore it may be an upstream regulatory gene of PTEN. Furthermore, it has previously been demonstrated that miRNA-214 is able to regulate PTEN in the processes of tumor invasion and metastasis in human gastric cancer, breast cancer and cervical cancer (51-53).

In the present study, a significant increase of miRNA-214 levels was observed in the tumor tissues, blood and saliva of patients with gingival carcinoma. These results suggest that PTEN in gingival carcinoma is directly downregulated by miRNA-214, as the upregulation of miRNA-214 may promote cleavage of PTEN, thus inhibiting the proapoptotic effect of PTEN protein, the formation of gingival cysts and ultimately gingival tumorigenesis.

In conclusion, the balance between miRNA-214 and PTEN levels may regulate the tumorigenesis and progression of gingival carcinoma. Additionally, miRNA-214 is more stable than PTEN in blood and saliva, and may be a more suitable marker for the early diagnosis of gingival carcinoma.

\section{Acknowledgements}

The authors of the present study want to thank Dr Shenglan Wang from the Oncology Department of the First People's Hospital of Yunnan Province (Kunming, China).

\section{References}

1. Qiu W: Oral and maxillofacial surgery. 6th edition. People's Medical Publishing House, Beijing, pp274-275, 2008.

2. Girdler NM: Oral ulceration: Benign or malignant? A diagnostic dilemma. Br Dent J 168: 386, 1990.

3. Ferlito A, Shaha AR and Rinaldo A: The incidence of lymph node micrometastases in patients pathologically staged No in cancer of oral cavity and oropharynx. Oral Oncol 38: 3-5, 2002.

4. Kademani D: Oral cancer. Mayo Clin Proc 82: 878-887, 2007.

5. Becker MT, Shores CG, Yu KK and Yarbrough WG: Molecular assay to detect metastatic head and neck squamous cell carcinoma. Arch Otolaryngol Head Neck Surg 130: 21-27, 2004.

6. Németh Z, Velich N, Bogdan S, Ujpál M, Szabó G and Suba ZS: The prognostic role of clinical, morphological and molecular markers in oral squamous cell tumors. Neoplasma 52: 95-102, 2005.

7. He H, Huang J, Ping F, Chen G, Zhang S and Dong $Y$ : Anatomical and clinical study of lingual arterial chemoembolization for tongue carcinoma. Oral Surg Oral Med Oral Pathol Oral Radiol Endod 103: e1-e5, 2007.

8. Mano H: Discovery and clinical application of mutations in the cancer genome. Nihon Rinsho 73: 1251-1255, 2015 (In Japanese).

9. Ohtsuka M, Ling H, Doki Y, Mori M and Calin GA: MicroRNA processing and human cancer. J Clin Med 4: 1651-1667, 2015.

10. Li JD, Liang RY, Zhao YP and Xu YL: Correlation analysis of VEGF and PTEN expression in gingival carcinoma. Shanghai Kou Qiang Yi Xue 23: 619-623, 2014 (In Chinese).

11. Boldrup L, Coates PJ, Wahlgren M, Laurell G and Nylander K: Subsite-based alterations in miR-21, miR-125b, and miR-203 in squamous cell carcinoma of the oral cavity and correlation to important target proteins. J Carcinog 11: 18, 2012.
12. Zhang KC, Xi HQ, Cui JX, Shen WS, Li JY, Wei B and Chen L: Hemolysis-free plasma miR-214 as novel biomarker of gastric cancer and is correlated with distant metastasis. Am J Cancer Res 5: 821-829, 2015.

13. Wang F, Lv P, Liu X, Zhu M and Qiu X: microRNA-214 enhances the invasion ability of breast cancer cells by targeting p53. Int J Mol Med 35: 1395-1402, 2015.

14. Wang J, Zhang X, Wang L, Yang Y, Dong Z, Wang H, Du L and Wang C: MicroRNA-214 suppresses oncogenesis and exerts impact on prognosis by targeting PDRG1 in bladder cancer. PLoS One 10: e0118086, 2015.

15. Wan DY, Zhang Z and Yang HH: Cardioprotective effect of miR-214 in myocardial ischemic postconditioning by down-regulation of hypoxia inducible factor 1, alpha subunit inhibitor. Cell Mol Biol (Noisy-le-grand) 61: 1-6, 2015.

16. Izawa T, Horiuchi T, Atarashi M, Kuwamura M and Yamate J: Anti-fibrotic role of miR-214 in Thioacetamide-induced liver cirrhosis in rats. Toxicol Pathol 43: 844-851, 2015.

17. Squarize CH, Castilho RM and Santos Pinto D Jr: Immunohistochemical evidence of PTEN in oral squamous cell carcinoma and its correlation with the histological malignancy grading system. J Oral Pathol Med 31: 379-384, 2002.

18. Li DM and Sun H: PTEN/MMAC1/TEP1 suppresses the tumorigenicity and induces G1 cell cycle arrest in human glioblastoma cells. Proc Natl Acad Sci USA 95: 15406-15411, 1998.

19. Di Cristofano A and Pandolfi PP: The multiple roles of PTEN in tumor suppression. Cell 100: 387-390, 2000.

20. Stiles B, Gilman V, Khanzenzon N, Lesche R, Li A, Qiao R, Liu X and $\mathrm{Wu} \mathrm{H}$ : Essential role of AKT-1/protein kinase B in PTEN-controlled tumorigenesis. Mol Cell Biol 22: 3842-3851, 2002.

21. Davies MA, Koul D, Dhesi H, Berman R, McDonnell TJ, McConkey D, Yung WK and Steck PA: Regulation of Akt/PKB activity, cellular growth, and apoptosis in prostate carcinoma cells by MMAC/PTEN1. Cancer Res 59: 2551-2556, 1999.

22. Tamura M, Gu J, Takino T and Yamada KM: Tumor suppressor PTEN inhibition of cell invasion, migration, and growth: Differential involvement of focal adhesion kinase and p130Cas. Cancer Res 59: 442-449, 1999.

23. McCubrey JA, Steelman LS, Abrams SL, Lee JT, Chang F, Bertrand FE, Navolanic PM, Terrian DM, Franklin RA, D'Assoro AB, et al: Roles of the RAF/MEK/ERK and PI3K/ PTEN/AKT pathways in malignant transformation and drug resistance. Adv Enzyme Regul 46: 249-279, 2006.

24. Punnoose EA, Ferraldeschi R, Szafer-Glusman E, Tucker EK, Mohan S, Flohr P, Riisnaes R, Miranda S, Figueiredo I, Rodrigues DN, et al: PTEN loss in circulating tumor cells correlates with PTEN loss in fresh tumor tissue from castration-resistant prostate cancer patients. Br J Cancer 113: 1225-1233, 2015.

25. Li J, Yen C, Liaw D, Podsypanina K, Bose S, Wang SI, Puc J, Miliaresis C, Rodgers L, McCombie R, et al: PTEN, a putative protein tyrosine phosphatase gene mutated in human brain, breast, and prostate cancer. Science 275: 1943-1947, 1997.

26. Mutter GL, Lin MC, Fitzgerald JT, Kum JB, Baak JP, Lees JA, Weng LP and Eng C: Altered PTEN expression as a diagnostic marker for the earliest endometrial precancers. J Natl Cancer Inst 92: 924-930, 2000.

27. Duerr EM, Rollbrocker B, Hayashi Y, Peters N, Meyer-Puttlitz B, Louis DN, Schramm J, Wiestler OD, Parsons R, Eng C and von Deimling A: PTEN mutations in gliomas and glioneuronal tumors. Oncogene 16: 2259-2264, 1998.

28. Tsao H, Zhang X, Benoit E and Haluska FG: Identification of PTEN/MMAC1 alterations in uncultured melanomas and melanoma cell lines. Oncogene 16: 3397-3402, 1998.

29. Rizvi MM, Alam MS, Ali A, Mehdi SJ, Batra S and Mandal AK: Aberrant promoter methylation and inactivation of PTEN gene in cervical carcinoma from Indian population. J Cancer Res Clin Oncol 137: 1255-1262, 2011.

30. Yang XF, Xin Y and Mao LL: Clinicopathological significance of PTEN and caspase-3 expressions in breast cancer. Chin Med Sci J 23: 95-102, 2008.

31. Bowen KA, Doan HQ, Zhou BP, Wang Q, Zhou Y, Rychahou PG and Evers BM: PTEN loss induces epithelial-mesenchymal transition in human colon cancer cells. Anticancer Res 29: 4439-4449, 2009.

32. Ren W, Qiang C, Gao L, Li SM, Zhang LM, Wang XL, Dong JW, Chen C, Liu CY and Zhi KQ: Circulating microRNA-21 (MIR-21) and phosphatase and tensin homolog (PTEN) are promising novel biomarkers for detection of oral squamous cell carcinoma. Biomarkers 19: 590-596, 2014. 
33. Livak KJ and Schmittgen TD: Analysis of relative gene expression data using real-tie quantitative PCR and the 2(-Delta Delta C (T)) Method. Methods 25: 402-408, 2001.

34. León LE and Calligaris SD: Visualization and analysis of MiRNA-targets interactions networks. Methods Mol Biol 1509: 209-220, 2017.

35. Falconieri G, Luna MA, Pizzolitto S, DeMaglio G, Angione V and Rocco M: Eosinophil-rich squamous carcinoma of the oral cavity: A study of 13 cases and delineation of a possible new microscopic entity. Ann Diagn Pathol 12: 322-327, 2008.

36. Kalnins IK, Leonard AG, Sako K, Razack MS and Shedd DP Correlation between prognosis and degree of lymph node involvement in carcinoma of the oral cavity. Am J Surg 134: 450-454, 1977.

37. Parkin DM, Bray F, Ferlay J and Pisani P: Estimating the world cancer burden: Globocan 2000. Int J Cancer 94: 153-156, 2001.

38. Rautava J, Luukkaa M, Heikinheimo K, Alin J, Grenman R and Happonen RP: Squamous cell carcinomas arising from different types of oral epithelia differ in their tumor and patient characteristics and survival. Oral Oncol 43: 911-919, 2007.

39. Yoshida R, Nakayama H, Nagata M, Hirosue A, Tanaka T, Kawahara K, Nakagawa Y, Matsuoka Y, Sakata J, Arita H, et al: Overexpression of nucleostemin contributes to an advanced malignant phenotype and a poor prognosis in oral squamous cell carcinoma. Br J Cancer 111: 2308-2315, 2014.

40. Kirita T, Yamanaka Y, Imai Y, Yamakawa N, Aoki K, Nakagawa Y, Yagyuu $\mathrm{T}$ and Hasegawa M: Preoperative concurrent chemoradiotherapy for stages II-IV oral squamous cell carcinoma: A retrospective analysis and the future possibility of this treatment strategy. Int J Oral Maxillofac Surg 41: 421-428, 2012.

41. Cheng YS, Rees T and Wright J: A review of research on salivary biomarkers for oral cancer detection. Clin Transl Med 3: 3, 2014.

42. Steck PA, Pershouse MA, Jasser SA, Yung WK, Lin H, Ligon AH, Langford LA, Baumgard ML, Hattier T, Davis T, et al: Identification of a candidate tumour suppressor gene, MMAC1, at chromosome $10 q 23.3$ that is mutated in multiple advanced cancers. Nat Genet 15: $356-362,1997$.
43. Li DM and Sun H: PTEN, encoded by a candidate tumor suppressor locus, is a novel protein tyrosine phosphatase regulated by transforming growth factor beta. Cancer Res 57: 2124-2129, 1997.

44. Wang YF and Tang WP: Progress of PTEN gene and oral and maxillofacial tumors. Shi Yong Lin Chuang Yi Xue 11: 125-127, 2010.

45. Liu K, Liu S, Zhang W, Jia B, Tan L, Jin Z and Liu Y: miR-494 promotes cell proliferation, migration and invasion, and increased sorafenib resistance in hepatocellular carcinoma by targeting PTEN. Oncol Rep 34: 1003-1010, 2015.

46. Howe K: Extraction of miRNAs from formalin-fixed paraffin-embedded (FFPE) tissues. Methods Mol Biol 1509: 17-24, 2017.

47. Deng H, Liu Q, Wang X, Huang R, Liu H, Lin Q, Zhou X and Xing D: Quantum dots-labeled strip biosensor for rapid and sensitive detection of microRNA based on target-recycled nonenzymatic amplification strategy. Biosens Bioelectron 87: 931-940, 2017.

48. Rissland OS: The organization and regulation of mRNA-protein complexes. Wiley Interdiscip Rev RNA 8, 2017.

49. Chen K and Rajewsky N: The evolution of gene regulation by transcription factors and microRNAs. Nat Rev Genet 8: 93-103, 2007.

50. Lewis BP, Burge CB and Bartel DP: Conserved seed pairing, often flanked by adenosines, indicates that thousands of human genes are microRNA targets. Cell 120: 15-20, 2005.

51. Yang TS, Yang XH, Wang XD, Wang YL, Zhou B and Song ZS: MiR-214 regulate gastric cancer cell proliferation, migration and invasion by targeting PTEN. Cancer Cell Int 13: 68, 2013.

52. Schwarzenbach H, Milde-Langosch K, Steinbach B, Müller V and Pantel K: Diagnostic potential of PTEN-targeting miR-214 in the blood of breast cancer patients. Breast Cancer Res Treat 134: 933-941, 2012.

53. Yang H, Kong W, He L, Zhao JJ, O'Donnell JD, Wang J, Wenham RM, Coppola D, Kruk PA, Nicosia SV and Cheng JQ: MicroRNA expression profiling in human ovarian cancer: miR-214 induces cell survival and cisplatin resistance by targeting PTEN. Cancer Res 68: 425-433, 2008. 